\title{
Erratum zu: 13. ÖAG-Jahrestagung der Österreichischen Adipositas Gesellschaft, „Adipositas in Medizin und Gesellschaft: Prävention, Therapie und Perspektiven“, Schloss Seggau, 28.-29. September 2012 - Abstracts
}

Online publiziert: 8 December 2012

(C) Springer-Verlag Wien 2012

\section{Erratum zu: Wien Klin. Wochenschrift (2012) 124:655- 664. DOI: 10.1007/s00508-012-0243-9}

Folgendes Abstract konnte aufgrund eines Irrtums bedauerlicherweise nicht aufgenommen werden:

Erfahrungen mit einem ambulanten Kurzzeitprogramm zur initialen Gewichtsreduktion bei übergewichtigen Erwachsenen

Denise Schaffner, Andrea Stensitzky, Peter Deibert, Daniel König und Aloys Berg

Institut für Präventive Medizin (IPM ${ }^{\circledR}$ ), Freiburg, Deutschland

Fragestellung: Da in der Adipositastherapie Initialerfolge oftmals den Anschub für eine langfristige Gewichtsabnahme geben können, soll über Erfahrungen mit einem ambulanten Kurzzeitprogramm berichtet werden.

Methodik: Nach einem regionalen tv-Bericht zum Thema „Abnehmen mit Erfolg“ meldeten sich 55 übergewichtige und adipöse Erwachsene im IPM, um an einem 6-wöchigen Interventionsprogramm teilzunehmen. Nach der Eingangsuntersuchung konnten 48 Patienten $\left(49,9 \pm 13,9 \mathrm{~J}, 34,2 \pm 4,7 \mathrm{~m} / \mathrm{kg}^{2}\right)$ in das Programm eingeschlossen werden. Die Intervention erfolgte nach einem im IPM entwickelten Konzept, bestehend aus ausführlicher Beratung zur Energiebilanzierung, dem Angebot eines Mahlzeitenersatzes auf Sojabasis (Almased ${ }^{\circledR}, 2 \mathrm{x}$ täglich, standardisiert nach KG) und der Möglichkeit zur telefonischen Begleitung. 40 der 48 Patienten schlossen die Intervention ab und nahmen an einer Abschlussuntersuchung teil.

Ergebnisse: Bei vergleichbarem Übergewicht waren die weiblichen Completer jünger $(\mathrm{n}=23,46,2 \pm 14,1 \mathrm{~J})$ als die männlichen $(\mathrm{n}=17,55,0 \pm 12,1 \mathrm{~J})$ und zeigten auch weniger Begleiterkrankungen; deutlich älter war die Subgruppe (SG) der Patienten mit MS und T2DM $(n=12,60,8 \pm 5,9 \mathrm{~J})$. Alle Completer zeigten eine deutliche Gewichtsabnahme nach Intervention: weibl. 93,4 $\pm 14,9$ auf $88,8 \pm 14,9 \mathrm{~kg}(-5,3 \%)$, männl. 108,9 $\pm 12,0$ auf $102,8 \pm 13,3(-5,6 \%)$, SG $103,2 \pm 11,8$ auf $97,0 \pm 11,8(-6,0 \%)$. Die Gewichtsabnahme war mit einer signifikanten Reduktion der begleitenden Risikofaktoren begleitet, besonders ausgeprägt für die SG-Patienten.

Schlussfolgerung: Die Ergebnisse zeigen, dass in einem standardisierten ambulanten Kurzzeitprogramm Männer wie Frauen erfolgreich an Gewicht verlieren und auch Patienten mit gestörter BZ-Regulation bereits nach 6-wöchiger Intervention erhebliche und für den weiteren Interventionsverlauf positive Erfolge erzielen können. 